\title{
Digital phenotyping of suicidal thoughts
}

\section{Citation}

Kleiman, Evan M., Brianna J. Turner, Szymon Fedor, Eleanor E. Beale, Rosalind W. Picard, Jeff C. Huffman, and Matthew K. Nock. 2018. "Digital Phenotyping of Suicidal Thoughts." Depression and Anxiety (April 10). doi:10.1002/da.22730.

\section{Published Version}

doi:10.1002/da.22730

\section{Permanent link}

http://nrs.harvard.edu/urn-3:HUL.InstRepos:37304564

\section{Terms of Use}

This article was downloaded from Harvard University's DASH repository, and is made available under the terms and conditions applicable to Open Access Policy Articles, as set forth at http:// nrs.harvard.edu/urn-3:HUL.InstRepos:dash.current.terms-of-use\#OAP

\section{Share Your Story}

The Harvard community has made this article openly available.

Please share how this access benefits you. Submit a story.

\section{Accessibility}


Running head: Digital Phenotyping of Suicidal Thoughts

IN PRESS: Depression and Anxiety

\title{
Digital Phenotyping of Suicidal Thoughts
}

Evan M. Kleiman, Ph.D., ${ }^{*}$ Brianna J. Turner, Ph.D., ${ }^{2}$ Szymon Fedor, Ph.D., ${ }^{3}$ Eleanor E. Beale, B.A., ${ }^{4,5}$ Rosalind W. Picard, Sc.D., ${ }^{3}$ Jeff C. Huffman, MD, ${ }^{4,5}$ \& Matthew K. Nock, Ph.D. ${ }^{1,4,6}$

\author{
${ }^{1}$ Harvard University, Department of Psychology. \\ ${ }^{2}$ University of Victoria, Department of Psychology. \\ ${ }^{3}$ Massachusetts Institute of Technology, Media Lab. \\ ${ }^{4}$ Massachusetts General Hospital, Department of Psychiatry. \\ ${ }^{5}$ Harvard Medical School, Department of Psychiatry. \\ ${ }^{6}$ Cambridge Computational Clinical Psychology Organization (C3PO).
}

\section{Acknowledgements}

The research was supported by the Chet and Will Griswold Suicide Prevention Fund (MKN), the Pershing Square Venture Fund for Research on the Foundations of Human Behavior (EMK), and the John D. and Catherine T. MacArthur Foundation (MKN).

\section{Corresponding author:}

Evan M. Kleiman

Department of Psychology, Harvard University

Email: ekleiman@fas.harvard.edu

Phone: 617-496-8563 


\begin{abstract}
Background: To examine whether there are subtypes of suicidal thinking using real-time digital monitoring, which allows for the measurement of such thoughts with greater temporal granularity than ever before possible.
\end{abstract}

Methods: We used smartphone-based real-time monitoring to assess suicidal thoughts four times per day in two samples: Adults who attempted suicide in the past year recruited from online forums ( $n=51$ participants with a total of 2,889 responses, surveyed over 28 days; ages ranged from $18-38$ years) and psychiatric inpatients with recent suicidal ideation or attempts $(n=32$ participants with a total of 640 responses, surveyed over the duration of inpatient treatment $[$ mean stay $=8.79$ days], ages ranged $23-68$ years). Latent profile analyses were used to identify distinct phenotypes of suicidal thinking based on the frequency, intensity, and variability of such thoughts.

Results: Across both samples, five distinct phenotypes of suicidal thinking emerged that differed primarily on the intensity and variability of suicidal thoughts. Participants whose profile was characterized by more severe, persistent suicidal thoughts (i.e., higher mean and lower variability around the mean) were most likely to have made a recent suicide attempt.

Conclusions: Suicidal thinking has historically been studied as a homogeneous construct, but using newly available monitoring technology we discovered five profiles of suicidal thinking. Key questions for future research include how these phenotypes prospectively relate to future suicidal behaviors, and whether they represent remain stable or trait-like over longer periods. 
The question of why people behave in ways that are harmful to themselves has puzzled scholars for thousands of years. The decision of whether to live or die has been called the "fundamental question of philosophy" (Camus, 1942) and has been the focus of scholarly work by most major philosophers throughout history (e.g., Kant, Sartre, Locke, Hume). In the sciences, the existence of suicidal thoughts and behaviors has presented a fundamental challenge to the belief that human and animal behavior is motivated by an innate and ever-present drive for self-preservation and gene survival (Dawkins, 1976; Lorenz, 1963; Wilson, 1978). Despite centuries of scholarly consideration and scientific investigation, key questions about suicide remain surprisingly unanswered, and it continues to be one of the leading causes of death worldwide. Indeed, approximately $9 \%$ of adults around the world report that they have seriously considered suicide at some point in their lives (Nock et al., 2008), and suicide accounts for over 800,000 deaths each year (World Health Organization, 2016a), more than all wars and other forms of interpersonal violence combined - meaning that we each are more likely to die by our own hand than by someone else's (World Health Organization, 2016b). More alarming is that suicide is projected to become an even greater contributor to the global burden of disease in the coming decades (Mathers \& Loncar, 2006). Understanding this perplexing aspect of human nature is one of the greatest challenges facing our society. Some of the key questions regarding suicide that remain unanswered involve understanding and classifying the everyday experience of individuals at risk for suicide.

In most areas of science (biology, chemistry, ethology, etc.), we obtain an understanding of phenomena of interest by directly observing and studying them (Kagan, 1967; Lorenz, 1981; Tinbergen, 1951, 1974). Historically, such an approach has not been possible in the study of suicidal thoughts and behaviors because they occur privately and episodically in a person's life, 
outside of the scope of standard psychological assessment methods. Moreover, the tools used to assess suicidal thoughts have been until now limited to intermittent assessments separated by weeks, months, or years, which does not address the fact that suicidal thoughts can be highly variable over a few hours (Bagge, Littlefield, Conner, Schumacher, \& Lee, 2014; Kleiman et al., 2017) and that suicide attempts can occur in response to rapidly escalating thoughts over time periods as short as a day (Millner, Lee, \& Nock, 2016). However, recent advances in smartphone-based real-time monitoring technology (i.e., ecological momentary assessment [EMA])(Shiffman, Stone, \& Hufford, 2008) have made it possible for the first time to overcome these limitations by allowing individuals to report on suicidal thoughts as they naturally occur in a variety of settings (e.g., over the course of clinical care, in a person's day-to-day life), which has been done in a small handful of studies to date (for review, see Kleiman \& Nock, 2018). Indeed, the use of smartphones and related portable devices are providing new opportunities for “digital phenotyping" that is, providing real-time characterization and quantification of human behavior in situ (Bidargaddi et al., 2017; Onnela \& Rauch, 2016; Torous, Onnela, \& Keshavan, 2017).

In two recent studies using real-time monitoring to observe suicidal thoughts, our group (Kleiman et al., 2017) and others (Hallensleben et al., 2017) found that thoughts of suicide vary considerably over short periods of time (e.g., hour to hour). Here we examine whether there are distinct profiles or subtypes of suicidal thinking by examining hour-to-hour changes in the reports of suicidal thoughts over the span of several weeks. Prior studies aimed at identifying subtypes of people at risk for suicide have done so using different profiles of risk factors (Bagge, Littlefield, \& Glenn, 2017; Ginley \& Bagge, 2017; Hamza \& Willoughby, 2013; Herres, Kodish, Fein, \& Diamond, 2017; Logan, Hall, \& Karch, 2011). 
Although several studies find different phenotypes based on risk factors for suicidal thoughts and several others have examined profiles of suicidal thinking based on the trajectory of suicidal thinking measured weeks or months apart (Czyz \& King, 2015; Goldston et al., 2016; Wolff et al., 2017), no prior studies have examined whether there are different phenotypes of suicidal thoughts based on the actual experience of thoughts itself. Recent theoretical work by Bernanke, Stanley, and Oquendo (2017) suggests that there may be at least two subtypes of the experience of suicidal thinking. One proposed subtype is characterized by large fluctuations in severity of suicidal thoughts in response to life stress. The other proposed subtype is characterized by persistent levels of suicidal thinking that do not fluctuate in response to life events. If such subtypes or classes of thoughts are observed, they may provide useful information for testing whether different phenotypes have different predictors, courses, and responsiveness to treatments, providing traction in areas of suicide research that have been relatively stagnant. Accordingly, the goal of this study was to see if we could classify phenotypes of suicidal thinking based upon the experience of suicidal thinking itself. To do this, we used data from two samples of people at high risk for suicide (recent attempters from the community and suicidal inpatients) who completed digital smartphone monitoring for 28 days (community members) or the length of inpatient treatment (inpatients).

\section{Method}

\section{Participants and recruitment}

Sample 1. The first sample consisted of 51 adults who had attempted suicide in the past year $(79 \%$ female, mean age $=23.59$ years, $S D=4.74$ years, range $18-38$ years $) .72 .54 \%$ of the sample self-identified as being of European decent, 9.80\% Hispanic, 7.84\% Asian, and the rest identified as another race. $96.07 \%$ of the sample had at least a high-school degree. Participants 
were recruited from forums relating to self-harm or suicide on the website Reddit

(www.reddit.com). Inclusion criteria included a suicide attempt with at least some intent to die occurring in the past year, fluency in English, age 18+ years, and regular access to an internetcapable smartphone. To determine eligibility, participants were first asked to complete a brief screener linked to the study ad. 854 people completed the screener, 103 of whom qualified for the study, 90 of whom were interested in the study (i.e., $87 \%$ of those who qualified). Of those who were interested and qualified, 54 people began the study $(60 \%$ of those who were interested and qualified), 51 (56\% of those who were interested and qualified) of whom completed at least three or more consecutive data points. Consecutive data points were required to calculate the variability statistics used in our analyses.

Sample 2. The second sample consisted of 32 adult inpatients who were hospitalized at the psychiatric inpatient unit at Massachusetts General Hospital for a recent suicide attempt or severe suicidal thoughts $(43.3 \%$ female, mean age $=42.53$ years, $S D=12.88$ years, range $23-$ 68 years). $81.25 \%$ of the sample self-identified as being of European decent, $6.25 \%$ Hispanic, $6.25 \%$ Asian, and the rest identified as another race. Inclusion criteria were admission due to a suicide attempt or severe suicidal thoughts and fluency in English (we loaned smartphones to those who did not have or own one). A study staff member approached any new admissions from the previous day that met inclusion criteria. 53 patients were approached during the study period, $41(77.3 \%)$ of whom were willing to participate and were consented for the study. Of those consented, 32 were included in the study ( $78 \%$ of those consented). Reasons for not being were as follows: one potential participant was discharged unexpectedly early, two did not appear to understand the study, two did not answer any of the smartphone surveys, and four did not complete three or more consecutive responses. 


\section{Procedure}

In both studies, participants completed a brief set of baseline questionnaires followed by a period of smart-phone based real-time monitoring where they were prompted to report on any experiences of suicidal thoughts four times per day each day (other experiences that were reported, such as mood states, are not relevant to the present study). Prompts were sent randomly within pre-defined intervals (i.e., times when the participant was awake). Below, we discuss areas of difference between the two studies.

Sample 1. Participants in the first sample completed real-time monitoring for a 28-day period. Prompts were delivered and responses collected via Mobile EMA software (www.ilumivu.com), which is compatible with both Android and iPhone smartphones. Participants were compensated with a $\$ 40$ (USD) gift card to Amazon.com, with a $\$ 10$ bonus for completing more than $75 \%$ of the prompts.

Sample 2. Participants in the second sample completed real-time monitoring for the duration of their inpatient stay (mean stay $=8.79$ days, $S D=8.23$ days, range $=2-46$ days, median $=7$ days). Prompts were delivered and responses collected via MovisensXS EMA software (www.movisens.com). MovisensXS is only compatible with Android smartphones, so we lent compatible phones to participants for the duration of the study if they did not have access to a smartphone, or if they owned an incompatible phone. Participants were compensated with $\$ 10$ (paid in cash) for each day they were in the study.

\section{Measures}

Baseline. Participants completed a brief demographics screener and the self-report version of the Self-Injurious Thoughts and Behaviors Interview (SITBI; Nock, Holmberg, Photos, \& Michel, 2007). The SITBI was used to assess suicide history (i.e., age of first attempt, 
number of lifetime attempts, date of most recent suicide attempt) and to also confirm our main inclusion criterion (past year suicide attempt).

Real-time Monitoring. At each prompt, participants were presented with three items assessing: (1) the desire to die by suicide ("How intense is your desire to kill yourself right now?"), (2) the intention to die by suicide ("How strong is your intention to kill yourself by suicide right now?"), and (3) the ability to resist the urge to die by suicide ("How strong is your ability to resist the urge to kill yourself right now?" which was reverse-coded). Each item for the first sample was on a 0 (not strong [intense] at all) to 4 (very strong [intense]) scale. Each item for the second sample was on a 0 (not strong [intense] at all) to 9 (very strong [intense]) scale. Since the items were on different scales, we could not combine samples. The three items were summed such that higher scores equaled more severe suicidal thoughts. These three component items were strongly intercorrelated in both samples $(|r|$ range from $.54-.89$, all $p<.001)$.

\section{Analytic strategy}

We used latent profile analysis (LPA) to test for the existence of distinct profiles of suicidal thinking in the real-time monitoring data described above. LPA is useful to classify groups of individuals based upon a set of continuous criterion variables, creating phenotypes of suicidal thoughts. There are several guidelines to assess the correct number of profiles: The Bayesian information criterion $(B I C)$, a measure of model fit; entropy, a measure of precision of classification of individuals into profiles; and the bootstrapped $k$-1 likelihood ratio test (LRT), a measure that compares a model with the current number $(k)$ of profiles to one with one fewer $(k$ 1) profile to determine if precision is improved by the addition of an extra profile. The optimal solution is indicated by the model with the lowest $B I C$, largest entropy, and a significant $k-1$ test. We used as indicators several measurements that quantify the patterns of suicidal thoughts 
experienced by each individual: (1) Mean scores across the three suicidal thoughts items, indicating average severity of suicidal thoughts across the study, (2) within-person standard deviations of each person's suicidal thought scores, indicating average within-person dispersion of suicidal thoughts around the mean, (3) the maximum total score for suicidal thought items, indicating the greatest intensity of suicidal thoughts experienced during the monitoring period, (4) the percent of prompts for which a non-zero score on suicidal thoughts was reported, indicating relative frequency of suicidal thinking, and (5) the root mean square of successive differences (RMSSD; von Neumann, Kent, Bellinson, \& Hart, 1941), indicating the average stability (or magnitude of change) of scores from one measurement occasion to the next. The $R M S S D$ is ideal to quantify non-linear variability in repeated measures and is interpreted such that larger values equal more variability from one point to the next and, if graphed, would correspond to a more jagged pattern. After we conducted the LPA, we examined if the phenotypes differed on suicide history variables using ANOVAs or chi-square tests. The LPA was performed in Mplus version 7 (Muthén \& Muthén, 2014). All other analyses were performed and figures were created in R (R Core Team, 2016).

\section{Results}

Participants in Sample 1 completed a total of 2,889 responses $(M=56.59$ responses per participant, $S D=37.77$ ) across a total of 1,311 days for an average of 2.20 responses per participant, per day (out of four possible prompts; $55.0 \%$ response rate). Participants in Sample 2 completed a total of 640 responses $(M=20$ responses per participant, $S D=17.48)$ across a total of 281 days for an average of 2.28 responses per participant, per day (out of four possible prompts; $57.0 \%$ response rate). 


\section{Subtypes of Suicidal Thinking (Sample 1)}

Table 1 contains fit statistics for all profiles. The LPA converged on a five-profile model $(B I C=1153.27$, entropy $=.961, k-1 L R T=58.95, p<.001)$. Although the six-profile model had a lower $B I C$ and higher entropy than the five-profile model, as well as a significant $k-1 L R T$ value, the five-profile model was still preferable for a few reasons. First, the six-profile model caused Mplus to produce non-identification warnings that likely indicated a model with too many profiles. Second, the six-profile model produced very small cell sizes (e.g., one phenotype profile had only two participants). Third, although there was an improvement in model fit from the five-profile to six-profile model, the increment in model fit was relatively smaller than the increment in fit seen in other models (e.g., entropy increased by .012 from the five-profile to sixprofile model, but increased by .040 from the four-profile model to the five-profile model). Thus, on balance, the five-profile model was preferable. Examination of the data from individual participants classified by these five profiles (Figure 1, Table 2) reveals that they are distinguished by their differences in mean and variation around that mean: (1) low mean, low variability; (2) low mean, moderate variability; (3) moderate mean, high variability; (4) high mean, low variability; and (5) high mean, high variability. Statistical comparisons of these five groups revealed no differences among these five phenotypes in terms of age of first suicide attempt and number of lifetime suicide attempts (Table 1). However, the phenotype typified by high mean and low variability (Phenotype 4) has a substantially higher proportion of individuals who had attempted suicide in the month before the study (and contained the one participant who attempted suicide in the week before the study) than any other phenotype. The phenotype typified by high mean and high variability (Phenotype 5) contained zero members who attempted suicide in the month before the study. Taken together, this indicates that among those who had 
higher mean levels of suicidal thoughts, a lower degree of variation around that mean was indicative of someone who had more recently attempted suicide.

\section{Results from Replication Sample (Sample 2)}

The LPA again converged on a five-profile model $(B I C=874.43$, entropy $=.991, k-1$ $L R T=34.37, p<.001)$. Table 1 contains fit statistics for all profiles. Examination of the data from individual participants classified by these five profiles revealed the same pattern of findings observed in Sample 1 in terms of differences in mean and variability of suicidal thinking and in group differences in history of suicide attempts (Table 2), with the only difference being that the higher rate of recent suicide attempt in Phenotype 4 was not significantly higher than all other groups in this sample. Figure 2 shows a visual depiction of the profiles, which looked similar to those from Sample 1.

\section{Discussion}

Suicide is among the leading causes of death worldwide and nearly $10 \%$ of people report thinking about suicide at some point in their lives. Unfortunately, our understanding of suicide and ability to predict and prevent it - has been hindered by a lack of information about the basic nature of suicidal thoughts. Using smartphone-based digital phenotyping methods, we identified five distinct phenotypes of suicidal thinking - a pattern that replicated across two samples with different levels of acuity (i.e., community-dwelling adults vs. psychiatrically hospitalized inpatients). The phenotypes differed primarily in the average severity of suicidal thoughts across measurement occasions, and the magnitude of within-person variability around that average. Moreover, the phenotype typified by more severe (i.e., higher mean) and more stable (i.e., lower $R M S S D$ ) suicidal ideation contained the highest proportion of individuals who had attempted suicide in the past month in the community sample. This finding was not statistically significant 
in the second sample, although the higher acuity of the sample may have resulted in a ceiling effect to detect group differences in recency of suicide attempts. Recent real-time monitoring studies have shown that there is substantial heterogeneity in the experience of suicidal thoughts (Kleiman et al., 2017), and the current study extends this earlier work by showing that there is order to this heterogeneity. Overall, these findings have important implications for future research aimed at better understanding the phenomenology of suicidal thinking and for clinical treatment of suicidal individuals.

This work is generally in line with Bernanke, Stanley, and Oquendo's (2007) proposal that there are two distinct phenotypes of suicidal thinking, typified by high versus low levels of variability. Although we found five phenotypes, one of the clearest descriptors of the phenotypes was how variable suicidal thinking was. Thus, it might be that the inclusion of other factors like mean level of suicidal thinking adds nuance to Bernake et al.'s theorized phenotypes (and indeed, the authors noted that more than two phenotypes might be a possibility). Relatedly, it may be that there are two superordinate phenotypes (high vs. low variability) and the other phenotypes we found describe sub-types of these superordinate phenotypes. One way in which our study could not fully test Bernake et al.'s model is that we did not have a measure of stress that allowed us to see if the peaks in suicidal thinking among those in the high variability phenotypes were predicted by stress. Additionally, our findings are also in line with a line of studies showing that more stable suicidal thinking is associated with increased risk for suicidal behavior. For example, one study found that among military servicemembers who reported having thoughts of suicide, those who had episodes of suicidal thinking lasting five hours or longer were at more than double the risk of those with shorter duration episodes of suicidal thinking to act on their suicidal thoughts (Nock et al., in press). 
These findings should be viewed in the context of several limitations. First, although the number of participants in each of the five phenotypes was evenly distributed in both samples, the samples were relatively small leading to somewhat small absolute numbers of participants per class. The replication of findings across two different samples increases confidence in the findings, but additional confirmatory studies are needed. Second, we followed participants in each sample for no more than one month at a time and so in this study we were unable to assess if one phenotype versus another predicts suicidal behaviors or other outcomes in the future. We would expect the more severe phenotypes (i.e., those with more severe/intense suicidal thoughts) to be at greater risk for another suicide attempt; however, future studies using longer-term follow-up periods are needed to test this. Third, these profiles may be influenced by the characteristics of the samples in which they were collected. For example, the inpatient in Sample 2 may have been less inclined to disclose severe suicidal thinking out of concerns of further clinical intervention or that they would not be released from the hospital. Thus, further replication in larger and more diverse samples is needed. Fourth, although there were many similarities between the two samples, there may have been important differences in the how participants interacted with the two software packages. For example, due to differences in how items were displayed on the screen, the software used in Sample 1 used a five-point scale for items whereas the software used in Sample 2 used a ten-point scale. Given that some work finds that data from scales with more points tend to be more normally-distributed (Leung, 2011), it may be that the ten-point scale used in Sample 2 was better equipped to detect variability in suicidal thinking. Finally, a broader issue that extends beyond the scope of this manuscript is the choice of terminology used to address the subtypes of suicidal thinking. Here, we used "digital phenotype" as it most closely matches the work on which this manuscript is based (Onnela \& 
Rauch, 2016; Torous et al., 2017) although other terminology may also apply here (e.g., "digital footprint"; Bidargaddi et al., 2017).

Although this study represents a first step towards developing a digital phenotype, there are several important directions for future studies. First, although we were primarily interested in quantifying variability and seeing if meaningful phenotypes could be established from this quantification, there may be additional ways to look at these data. For example, growth mixture modelling would allow future studies to assess whether individuals have different trajectories of change in suicidal ideation (e.g., over the inpatient or post-discharge period). Second, future studies should explore the full range of available data (e.g., GPS data, call logs) that may further refine phenotypes of suicidal ideation (Kleiman \& Nock, 2017). Third, in both samples we conceptualized suicidal thinking with a three-item composite that assessed one's desire to die by suicide, intent to die by suicide, and ability to resist the urge to die by suicide. However, additional conceptualizations of suicidal thinking have been linked to future suicidal behavior (e.g., persistence of suicidal thoughts; controlability of suicidal thoughts; Nock et al., in press). Accordingly, future studies should examine whether other conceptualizations of suicidal thinking help to further distinguish phenotypes of suicidal thinking. Finally, studies with longer follow-up lengths may help assess stability or change in phenotypes. If studies with longer follow-up periods find that some individuals' phenotype membership changes over time, it may be useful to examine factors that predict these changes.

Clinically, these findings suggest that it is important to not just assess clients' average level of suicidal thinking but also variability around that average. This is important because those who have had severe and stable suicidal thinking (as in the high mean/low variability phenotype in this study) may be at greater risk of engaging in suicidal behavior, although future studies are 
needed to fully test this idea. In conclusion, the results from this initial study suggest that realtime monitoring of suicidal individuals is feasible and can provide valuable new information about suicidal thoughts - supporting the conduct of future, longer-term digital phenotyping studies of suicidal behavior. 


\section{Conflict of interest statement}

The authors have no conflicts of interest to report. 


\section{References}

Bagge, C. L., Littlefield, A. K., Conner, K. R., Schumacher, J. A., \& Lee, H.-J. (2014). Nearterm predictors of the intensity of suicidal ideation: An examination of the $24 \mathrm{~h}$ prior to a recent suicide attempt. Journal of Affective Disorders, 165, 53-58.

https://doi.org/10.1016/j.jad.2014.04.010

Bagge, C. L., Littlefield, A. K., \& Glenn, C. R. (2017). Trajectories of affective response as warning signs for suicide attempts: An examination of the 48 hours prior to a recent suicide attempt. Clinical Psychological Science, 2167702616681628.

https://doi.org/10.1177/2167702616681628

Bernanke, J. A., Stanley, B. H., \& Oquendo, M. A. (2017). Toward fine-grained phenotyping of suicidal behavior: The role of suicidal subtypes. Molecular Psychiatry. https://doi.org/10.1038/mp.2017.123

Bidargaddi, N., Musiat, P., Makinen, V.-P., Ermes, M., Schrader, G., \& Licinio, J. (2017). Digital footprints: Facilitating large-scale environmental psychiatric research in naturalistic settings through data from everyday technologies. Molecular Psychiatry, 22(2), 164-169. https://doi.org/10.1038/mp.2016.224

Camus, A. (1942). The Myth of Sisyphus and Other Essays. (J. O’Brien, Trans.). New York: Vintage.

Czyz, E. K., \& King, C. A. (2015). Longitudinal trajectories of suicidal ideation and subsequent suicide attempts among adolescent inpatients. Journal of Clinical Child and Adolescent Psychology: The Official Journal for the Society of Clinical Child and Adolescent Psychology, American Psychological Association, Division 53, 44(1), 181-193. https://doi.org/10.1080/15374416.2013.836454 
Dawkins, R. (1976). The Selfish Gene. New York, NY: Oxford University Press.

Ginley, M. K., \& Bagge, C. L. (2017). Psychiatric heterogeneity of recent suicide attempters: A latent class analysis. Psychiatry Research, 251, 1-7. https://doi.org/10.1016/j.psychres.2017.02.004

Goldston, D. B., Erkanli, A., Daniel, S. S., Heilbron, N., Weller, B. E., \& Doyle, O. (2016). Developmental Trajectories of Suicidal Thoughts and Behaviors From Adolescence Through Adulthood. Journal of the American Academy of Child and Adolescent Psychiatry, 55(5), 400-407.e1. https://doi.org/10.1016/j.jaac.2016.02.010

Hallensleben, N., Spangenberg, L., Forkmann, T., Rath, D., Hegerl, U., Kersting, A., ... Glaesmer, H. (2017). Investigating the dynamics of suicidal ideation: preliminary findings from a study using ecological momentary assessments in psychiatric inpatients. Crisis, 1-5. https://doi.org/10.1027/0227-5910/a000464

Hamza, C. A., \& Willoughby, T. (2013). Nonsuicidal self-injury and suicidal behavior: A latent class analysis among young adults. PLOS ONE, 8(3), e59955. https://doi.org/10.1371/journal.pone.0059955

Herres, J., Kodish, T., Fein, J., \& Diamond, G. (2017). Screening to identify groups of pediatric emergency department patients using latent class analysis of reported suicidal ideation and behavior and non-suicidal self-injury. Archives of Suicide Research, Epub ahead of print, 1-12. https://doi.org/10.1080/13811118.2017.1283264

Kagan, J. (1967). On the need for relativism. American Psychologist, 22(2), 131-142. https://doi.org/10.1037/h0024311 
Kleiman, E. M., \& Nock, M. K. (2017). Advances in scientific possibilities offered by real-time monitoring technology. Psychiatry: Interpersonal and Biological Processes. https://doi.org/10.1080/00332747.2017.1325661

Kleiman, E. M., \& Nock, M. K. (2018). Real-time assessment of suicidal thoughts and behaviors. Current Opinion in Psychology, 22, 33-37. https://doi.org/10.1016/j.copsyc.2017.07.026

Kleiman, E. M., Turner, B. J., Fedor, S., Beale, E. E., Huffman, J. C., \& Nock, M. K. (2017). Examination of real-time fluctuations in suicidal ideation and its risk factors: Results from two ecological momentary assessment studies. Journal of Abnormal Psychology. https://doi.org/10.1037/abn0000273

Leung, S.-O. (2011). A comparison of psychometric properties and normality in 4-, 5-, 6-, and 11-point likert scales. Journal of Social Service Research, 37(4), 412-421. https://doi.org/10.1080/01488376.2011.580697

Logan, J., Hall, J., \& Karch, D. (2011). Suicide categories by patterns of known risk factors: A latent class analysis. Archives of General Psychiatry, 68(9), 935-941. https://doi.org/10.1001/archgenpsychiatry.2011.85

Lorenz, K. Z. (1963). On Aggression. New York: Harcourt Brace.

Lorenz, K. Z. (1981). The Foundations of Ethology. Vienna: Springer.

Mathers, C. D., \& Loncar, D. (2006). Projections of global mortality and burden of disease from 2002 to 2030. PLOS Medicine, 3(11), e442.

https://doi.org/10.1371/journal.pmed.0030442 
Millner, A. J., Lee, M. D., \& Nock, M. K. (2016). Describing and measuring the pathway to suicide attempts: A preliminary study. Suicide and Life-Threatening Behavior, epub ahead of print. https://doi.org/10.1111/sltb.12284

Muthén, L. K., \& Muthén, B. O. (2014). Mplus User's Guide. Seventh Edition. Los Angeles, CA: Muthén \& Muthén.

Nock, M. K., Borges, G., Bromet, E. J., Alonso, J., Angermeyer, M., Beautrais, A., ... Williams, D. R. (2008). Cross-national prevalence and risk factors for suicidal ideation, plans, and attempts. British Journal of Psychiatry, 192, 98-105. https://doi.org/10.1192/bjp.bp.107.040113

Nock, M. K., Holmberg, E. B., Photos, V. I., \& Michel, B. D. (2007). Self-Injurious Thoughts and Behaviors Interview: Development, reliability, and validity in an adolescent sample. Psychological Assessment, 19(3), 309-317. https://doi.org/10.1037/1040-3590.19.3.309

Nock, M. K., Millner, A. J., Joiner, T. E., Gutierrez, P. M., Han, G., Hwang, I., ... Kessler, R. C. (in press). Risk factors for the transition from suicide ideation to suicide attempt: Results from the Army Study to Assess Risk and Resilience in Servicemembers (Army STARRS). Journal of Abnormal Psychology.

Onnela, J.-P., \& Rauch, S. L. (2016). Harnessing smartphone-based digital phenotyping to enhance behavioral and mental health. Neuropsychopharmacology, 41(7), 1691-1696. https://doi.org/10.1038/npp.2016.7

R Core Team. (2016). R: A language and environment for statistical computing. Vienna, Austria: R Foundation for Statistical Computing. Retrieved from https:/www.R-project.org/ 
Shiffman, S., Stone, A. A., \& Hufford, M. R. (2008). Ecological momentary assessment. Annual Review of Clinical Psychology, 4, 1-32.

https://doi.org/10.1146/annurev.clinpsy.3.022806.091415

Tinbergen, N. (1951). The study of instinct (Vol. xii). New York, NY, US: Clarendon Press/Oxford University Press.

Tinbergen, N. (1974). Ethology and stress diseases. Science, 185(4145), 20-27. https://doi.org/10.1126/science.185.4145.20

Torous, J., Onnela, J.-P., \& Keshavan, M. (2017). New dimensions and new tools to realize the potential of RDoC: Digital phenotyping via smartphones and connected devices. Translational Psychiatry, 7(3), e1053. https://doi.org/10.1038/tp.2017.25

von Neumann, J., Kent, R. H., Bellinson, H. R., \& Hart, B. I. (1941). The mean square successive difference. The Annals of Mathematical Statistics, 12(2), 153-162.

Wilson, E. O. (1978). On Human Nature (Revised edition). Cambridge, Mass: Harvard University Press.

Wolff, J. C., Davis, S., Liu, R. T., Cha, C. B., Cheek, S. M., Nestor, B. A., ... Spirito, A. (2017). Trajectories of Suicidal Ideation among Adolescents Following Psychiatric Hospitalization. Journal of Abnormal Child Psychology. https://doi.org/10.1007/s10802017-0293-6

World Health Organization (Ed.). (2016a). Monitoring health for the SDGs: Sustainable development goals. Geneva: World Health Organization.

World Health Organization. (2016b). WHO Mortality Database. Retrieved from http://apps.who.int/healthinfo/statistics/mortality/whodpms/ 
Table 1.

Latent profile analyses fit statistics and phenotype profile membership

\section{Sample 1 (past-year attempters)}

\begin{tabular}{|c|c|c|c|c|c|c|c|c|c|}
\hline \multicolumn{4}{|c|}{$\underline{\text { Fit Statistics }}$} & \multicolumn{6}{|c|}{ Phenotype profile membership } \\
\hline Profiles & $B I C$ & Entropy & $k-1 L R T$ & 1 & 2 & 3 & 4 & 5 & 6 \\
\hline 2 & 1216.14 & .931 & $131.64, p<.001$ & $51.92 \%$ & $46.15 \%$ & & & & \\
\hline 3 & 1186.23 & .911 & $53.62, p<.001$ & $40.38 \%$ & $32.69 \%$ & $25.00 \%$ & & & \\
\hline 4 & 1172.32 & .921 & $37.59, p<.001$ & $28.85 \%$ & $28.85 \%$ & $23.08 \%$ & $17.31 \%$ & & \\
\hline 5 & 1153.27 & .961 & $58.95, p<.001$ & $28.85 \%$ & $26.92 \%$ & $17.31 \%$ & $17.31 \%$ & $7.69 \%$ & \\
\hline 6 & 1148.73 & .973 & $28.45 p<.001$ & $28.85 \%$ & $26.92 \%$ & $19.23 \%$ & $15.38 \%$ & $5.77 \%$ & $3.85 \%$ \\
\hline
\end{tabular}

Sample 2 (suicidal inpatients)

$\underline{\text { Fit Statistics }}$

Phenotype profile membership

\begin{tabular}{cccccccccc} 
Profiles & $B I C$ & Entropy & $k-1 L R T$ & 1 & 2 & 3 & 4 & 5 & 6 \\
\hline 2 & 939.56 & .916 & $69.31, p<.001$ & $50.00 \%$ & $50.00 \%$ & & & & \\
3 & 898.54 & .911 & $61.82, p<.001$ & $43.75 \%$ & $40.63 \%$ & $15.63 \%$ & & & \\
4 & 888.01 & .993 & $31.32, p<.001$ & $43.75 \%$ & $28.13 \%$ & $15.63 \%$ & $12.50 \%$ & & \\
5 & 874.43 & .991 & $34.37, p<.001$ & $28.13 \%$ & $28.13 \%$ & $18.75 \%$ & $12.50 \%$ & $12.50 \%$ & \\
6 & 872.52 & .993 & $22.28 p=.069$ & $28.13 \%$ & $25.00 \%$ & $15.63 \%$ & $12.50 \%$ & $12.50 \%$ & $6.25 \%$ \\
\hline \hline
\end{tabular}

Note: $B I C=$ Bayesian Information Criterion; $L R T=$ Likelihood Ratio Test. Profile numbers sorted by largest to smallest, but do not necessarily correspond to the same profile from model to model (e.g., profile 3 in the three-profile model is not the same exact profile as profile 3 in the four-profile model). 
Table 2. Comparison of phenotype profiles

\section{$\underline{\text { Sample } 1 \text { (past-year attempters) }}$}

\begin{tabular}{llllllll}
\multirow{2}{*}{ Profile } & $1(n=9)$ & $2(n=15)$ & $3(n=14)$ & $4(n=4)$ & $5(n=9)$ & Test & $p$ \\
& Low mean, & Low mean, & Mod mean, & High mean, & High mean, & stat. & $p$ \\
& Low variability & Mod. variability & High variability & Low variability & High variability & &
\end{tabular}

\section{Thoughts (LPA indicators)}

$\begin{array}{llllllll}R M S S D & 2.01_{\mathrm{b}} & 3.08_{\mathrm{c}} & 1.64_{\mathrm{a}, \mathrm{b}} & 3.38_{\mathrm{c}} & 45.38 & <.001 \\ \text { Mean } & 0.84_{\mathrm{a}} & 1.27_{\mathrm{a}} & 2.34_{\mathrm{b}} & 6.00_{\mathrm{c}} & 5.51_{\mathrm{c}} & 69.42 & <.001 \\ S D & 0.42_{\mathrm{a}} & 1.66_{\mathrm{b}} & 2.78_{\mathrm{c}} & 1.50_{\mathrm{b}} & 2.83_{\mathrm{c}} & 59.52 & <.001 \\ \operatorname{Max} & 0.68_{\mathrm{a}} & 6.27_{\mathrm{b}} & 9.93_{\mathrm{c}} & 9.50_{\mathrm{c}} & 11.22_{\mathrm{c}} & 57.82 & <.001 \\ \%>0 & 2.70_{\mathrm{a}} & 47.03_{\mathrm{a}, \mathrm{b}} & 58.56_{\mathrm{b}} & 99.94_{\mathrm{c}} & 94.96_{\mathrm{c}} & 21.13<.001\end{array}$

\section{Suicide history}

\begin{tabular}{|c|c|c|c|c|c|c|}
\hline Age of first attempt & 17.89 & 17.67 & 14.08 & 16.50 & 13.57 & 2.50 \\
\hline \# of lifetime attempts & 3.00 & 4.07 & 5.08 & 3.25 & 6.71 & 1.44 \\
\hline$\%$ past month attempt & $20 \%{ }_{\mathrm{a}}$ & $6.7 \% \mathrm{a}$ & $28.6 \% \mathrm{a}_{\mathrm{a}}$ & $75 \% \mathrm{a}_{\mathrm{a}}$ & $0 \%$ b & 11.57 \\
\hline$\%$ past week attempt & $0 \% \%_{a}$ & $0 \% \%_{a}$ & $0 \% \%_{a}$ & $25 \% \mathrm{ob}_{\mathrm{b}}$ & $0 \%{ }_{a}$ & 11.23 \\
\hline
\end{tabular}

Sample 2 (suicidal inpatients)

Profile

$1(n=4) \quad 2(n=9)$

$3(n=6) \quad 4(n=9)$

$5(n=4)$

High mean,

High mean,

Test

Low mean, Mod. mean,

Mod. mean,

Mod. variability

High variability

stat. $p$

\section{Thoughts (LPA indicators)}

$\begin{array}{lllllll}R M S S D & 0.69_{\mathrm{a}} & 1.49_{\mathrm{a}} & 4.09_{\mathrm{b}} & 3.63_{\mathrm{b}} & 6.81_{\mathrm{c}} & 63.56<.001 \\ \text { Mean } & 0.54_{\mathrm{a}} & 3.73_{\mathrm{b}} & 3.07_{\mathrm{a}, \mathrm{b}} & 8.47_{\mathrm{c}} & 12.00_{\mathrm{d}} & 19.92<.001 \\ S D & 0.83_{\mathrm{a}} & 1.22_{\mathrm{b}} & 3.40_{\mathrm{c}} & 3.29_{\mathrm{d}} & 5.12_{\mathrm{e}} & 39.78<.001 \\ \text { Max } & 2.50_{\mathrm{a}} & 6.33_{\mathrm{a}} & 9.83_{\mathrm{b}} & 15.00_{\mathrm{b}} & 23.00_{\mathrm{c}} & 29.40<.001 \\ \%>0 & 21.99_{\mathrm{a}} & 99.5_{\mathrm{b}} & 62.73_{\mathrm{c}} & 99.11_{\mathrm{b}} & 99.57_{\mathrm{b}} & 39.99<.001\end{array}$

\section{Suicide history}

$\begin{array}{llllllll}\text { Age of first attempt } & 21.50 & 43.33 & 29.20 & 24.33 & 23.00 & 2.20 & .399 \\ \text { \# of lifetime attempts } & 3.25 & 2.17 & 2.40 & 6.67 & 8.33 & 1.07 & .108 \\ \text { \% past month attempt } & 50.00 \% & 66.70 \% & 83.33 \% & 66.70 \% & 100.00 \% & 2.38 & .666 \\ \text { \% past week attempt } & 25.0 \% & 50.0 \% & 40.0 \% & 66.7 \% & 66.7 \% & 2.20 & .699\end{array}$

Note: Numbers in rows that do not share subscripts are significantly different at $p<.05 . F[4,47], F[4,27]$ for Study 1 and 2 ANVOAS, respectively. Df $=4$ for $\chi^{2}$ tests in both studies. 
Figure 1. Individual time series plots of suicidal thoughts in sample 1 (raw scores)
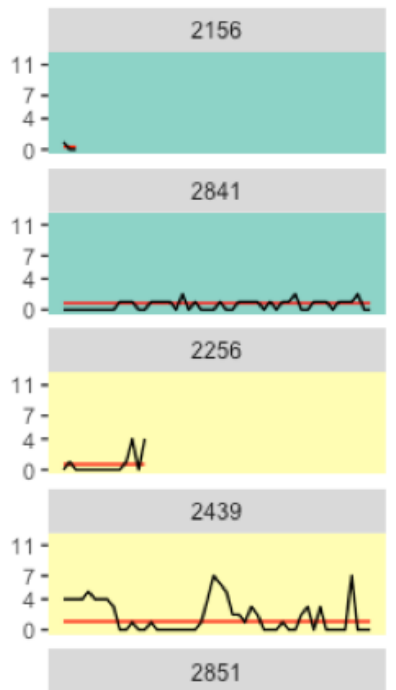

$11-$

:-

음
$\frac{0}{0}$
$\frac{0}{\pi}$
$\frac{0}{0}$
ต

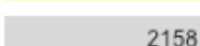

2158

Malatha 2416

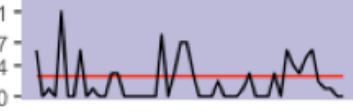

2848
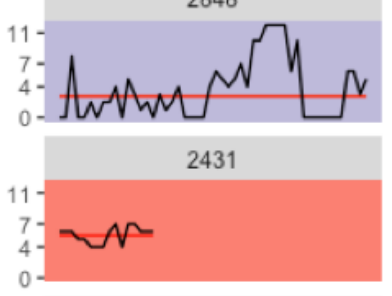

2418
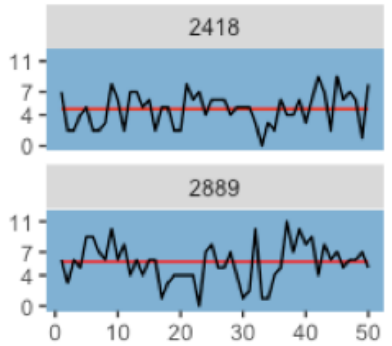
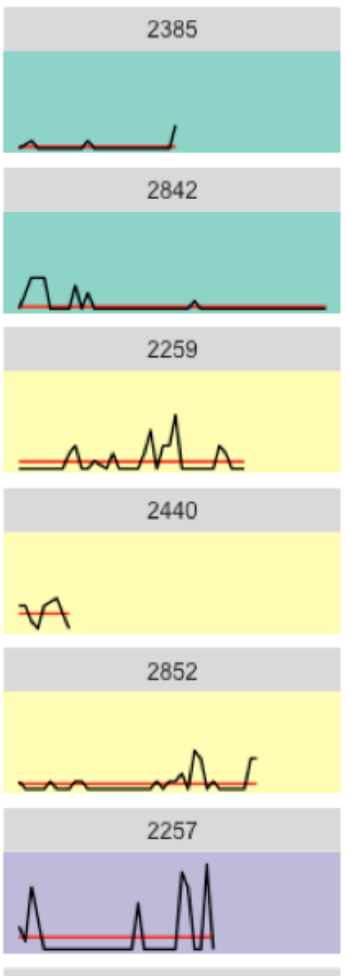

2417

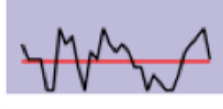

2888
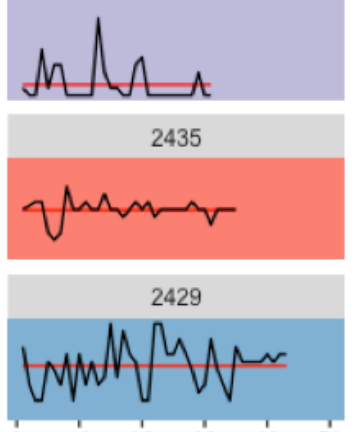

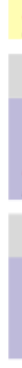
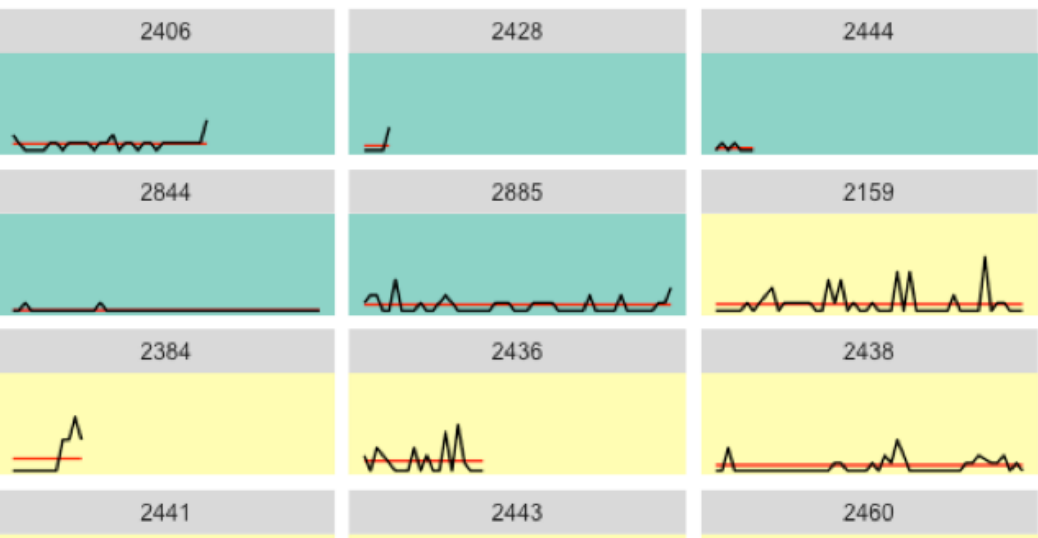

ANA
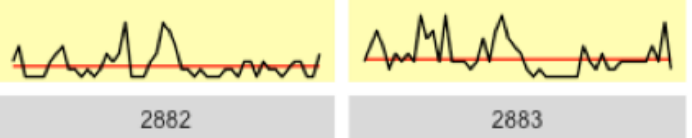

ompurath
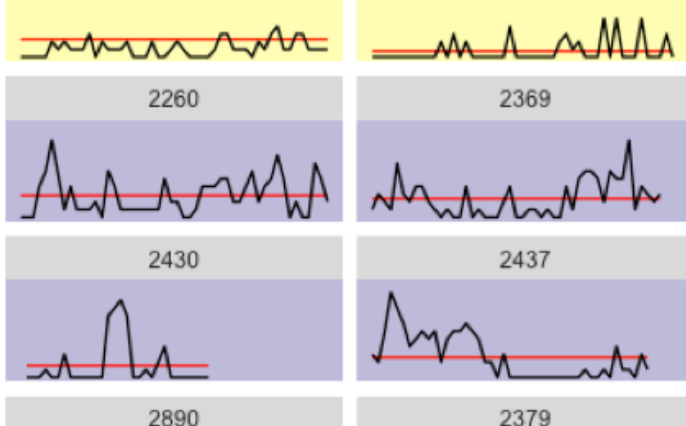

2890
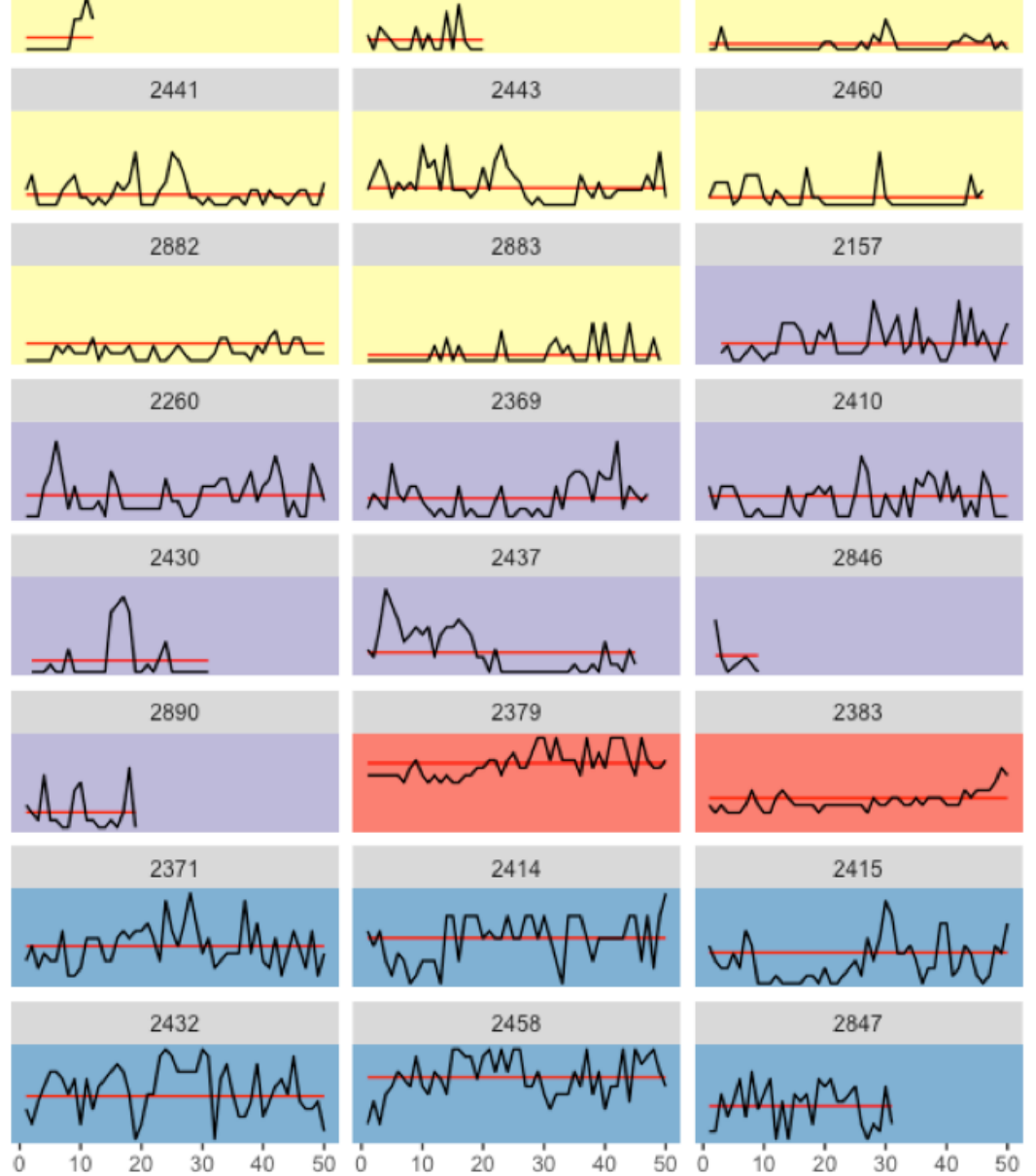

Phenotype profile

1: Low mean, low variability 2: 3 : Moderate mean, high variability - 4: High mean, low variability

Observation Number (approx 4-8 hours apart)

Note: Red line $=$ participants' mean scores. 
Figure 2. Individual time series plots of suicidal thoughts in sample 2 (raw scores)

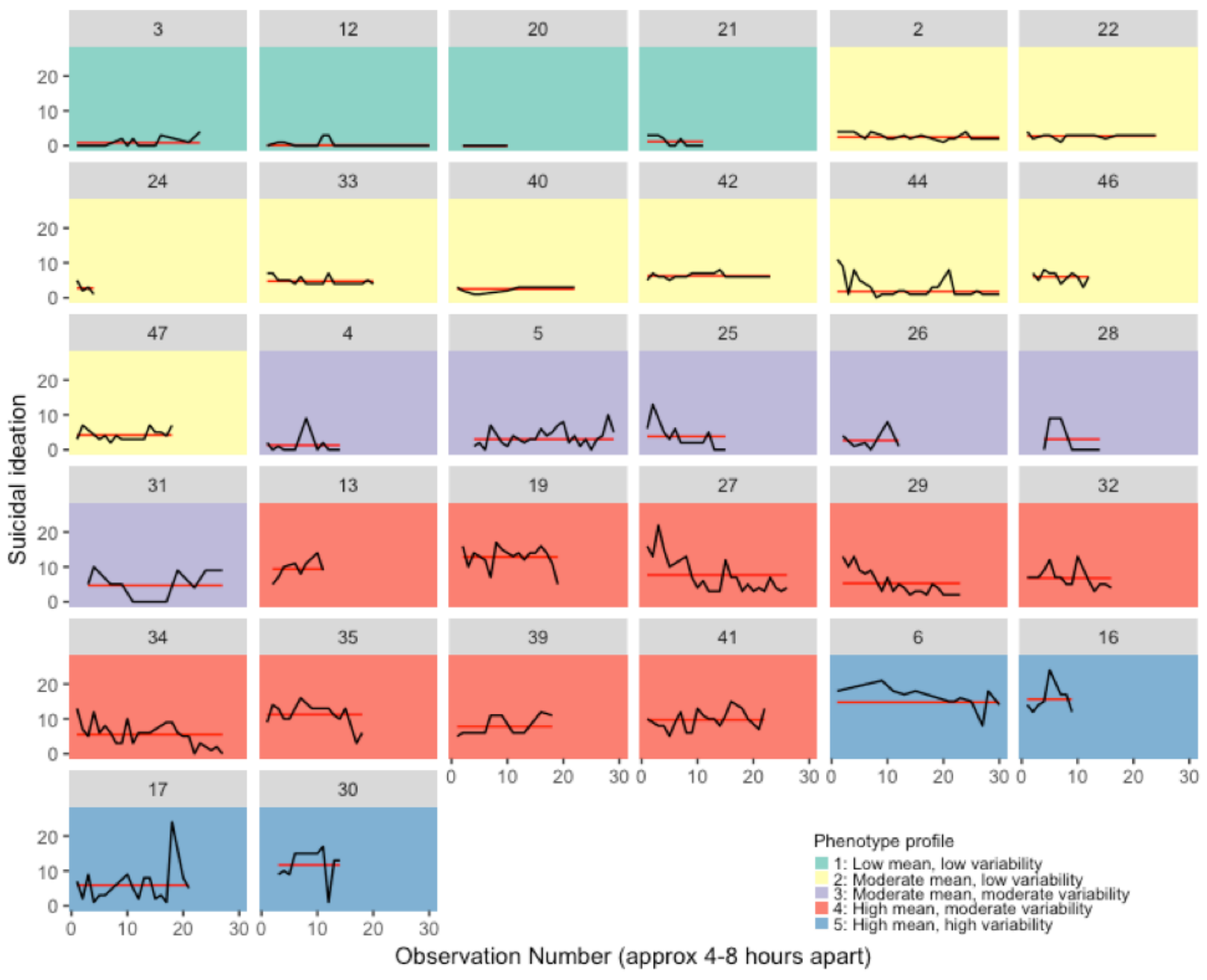

Note: Red line $=$ participants' mean scores. 